\title{
Prevalence and Etiology of Abnormal Liver Tests in an Adult Population in Jilin, China
}

\author{
Hong Zhang, Shu Mei He, Jie Sun, ChunYan Wang, Yan Fang Jiang, Qing Gu, Xiang Wei Feng, Bing Du, Wei \\ Wang, Xiao Dong Shi, Si Qi Zhang, Wan Yu Li, Jun Qi Niu ${ }^{凶}$
}

Department of Hepatology, First Hospital, Jilin University, Changchun 130021, China

$\triangle$ Corresponding author: Dr. Junqi Niu, Department of Hepatology, First Hospital, Jilin University, Changchun 130021, China; TEL: 86-431-85612708; FAX: 81-431-85612708; E-mail: junqiniu@yahoo.com.cn

(c) Ivyspring International Publisher. This is an open-access article distributed under the terms of the Creative Commons License (http://creativecommons.org/ licenses/by-nc-nd/3.0/). Reproduction is permitted for personal, noncommercial use, provided that the article is in whole, unmodified, and properly cited.

Received: 2011.01.18; Accepted: 2011.03.04; Published: 2011.03.25

\begin{abstract}
Background: Serum biochemical liver tests (LTs) (alanine aminotransferase, aspartate aminotransferase, and gamma glutamyltransferase) and platelet counts are often used to screen for chronic liver disease. We determined the prevalence and etiologies of abnormal LTs in an adult population in Jilin, China.

Methods: $A$ total of 3791 individuals between the ages of 18 and 79 years were interviewed and then underwent ultrasonography and blood tests.

Results: The prevalence of abnormal LTs was $14.77 \%$ (560 out of 379 I subjects). The risk factors for abnormal LTs were non-alcoholic fatty liver disease (NAFLD) alone, which accounted for $11.61 \%$, metabolic syndrome alone for $25 \%$, or both for $22.14 \%$. Abnormal LTs were more common in male than in female subjects. The development of abnormal LTs was correlated with older age males, increased daily alcohol intake, poor quality of sleep, smoking, fasting plasma glucose, body mass index, triglyceridemia, and low-density lipoprotein. Abnormal LTs in patients with metabolic syndrome and NAFLD were associated with high fasting plasma glucose, triglycerides, body mass index, low density lipoprotein, male, young age, poor sleep quality, smoking, and alcohol intake. However, abnormal LTs in patients with hepatitis B virus were associated with gender and increased age.

Conclusions: The results from the current study demonstrated that the prevalence of abnormal LTs is high in the population (14.77\%). Metabolic syndrome, NAFLD, and alcohol intake appear to be potentially important causes of the observed abnormal LTs.
\end{abstract}

Key words: alanine aminotransferase; metabolic syndrome; non-alcoholic fatty liver disease; ultrasonography; seroepidemiologic marker.

\section{Introduction}

Chronic liver disease (CLD) is a worldwide public health concern, which often contributes to end-stage liver disease. The risk factors for chronic liver disease include non-alcoholic fatty liver disease (NAFLD), hepatitis B virus (HBV) and hepatitis C virus (HCV), and alcohol consumption [1]. Due to increased unhealthy dietary habits and sedentary lifestyles, NAFLD, which is associated with insulin resistance [2], has emerged as a leading cause of chronic liver disease in Western countries [3-4]. The rapidly increasing appearance of metabolic disorders, including NAFLD, has also been recently reported in China [5]. Therefore, the main question is whether there is a similar change in the etiology of chronic 
liver disease in China. The increasing prevalence of obesity, coupled with diabetes, dyslipidemia, hypertension, and ultimately metabolic syndrome (MS) puts a very large population at risk of future liver failure $[4,6]$. Before the development of liver failure, the course of CLD is usually long and asymptomatic. However, in early stages, alanine aminotransferase (ALT), aspartate aminotransferase (AST), and gamma glutamyltransferase (GGT) may increase. Platelet count and indices of hepatic biosynthesis usually decrease in advanced stages of CLD. The prevalence and etiology of elevated serum aminotransferase levels as markers of CLD have been studied in blood donors, overweight adolescents, and liver disease patients [7-9]. But, the results of these studies are likely to have selection and referral biases. With regard to the general population, relevant data in the period 1988-1994 have been collected and published in the United States [10-11]. For northern China, where, as in other areas of Asian countries, eating habits differ from those in more industrialized areas, relevant data are still lacking on NAFLD, MS, and alcohol-induced liver damage. However, it has been shown that NAFLD is the commonest cause of elevated ALT and presumed liver injury in Taiwan [12]. A population-based survey of serum biochemical liver tests could provide a reliable tool to assess potential liver diseases, although liver biopsy is the gold standard for the diagnosis of fatty liver disease and hepatocellular damage. However, liver biopsy is an invasive, inconvenient, and expensive procedure, and carries a risk of complications, which is definitely not suitable for a population-based study. Other alternatives include ultrasonography and computer tomography, which provides an accurate diagnosis for a number of liver diseases, such as fatty liver. We assessed the prevalence and etiology of abnormal liver tests (LTs) in the general population of JiLin, China to evaluate the potential burden and causes of CLD.

\section{Materials and Methods \\ Design and study population}

A cross-sectional study of aminotransferase levels and the metabolic factors (triglyceridemia, body mass index, and low density lipoprotein et al) was carried out in Dehui, Jilin, China in 2007 (population approximately 410,600). Dehui is located $81 \mathrm{~km}$ from Changchun, the largest city in the area. Earnings of most inhabitants of Dehui are in the middle of the income range. The sex and age distribution of inhabitants are similar to those of JiLin in general. Therefore, Dehui City is representative of other areas in the province in terms of the level of life, economic and cultural development.

A two-stage, tiered-system sampling method was used. This survey was comprehensive and included geographic, economic, cultural, and other parameters. There are 308 villages and 51 neighborhood committees in Dehui. The first survey covered rural areas, and the second covered urban areas. Each stage was divided into two layers. In the first layer, the populations of the villages or neighborhood committees were sorted, and the villages or neighborhood committees were selected by a computer according to the principle of equidistant random samples of the population size. We selected 9 villages and 11 neighborhood committees. In the second layer, the households were marked by the distance from the centre of the villages or neighborhood committees, and they were selected according to the principle of equidistant random samples of the distance. Then, 150-200 or 80-100 households were computer selected in villages or neighborhood committees, respectively. A sample of the general population in the selected households consisting of individuals who were at least 18 years of age and had lived in the same area for more than 10 years was selected using a systematic random 1 in 3 sampling procedure from the census list, which had been updated on February 1, 2007.

According to the formula of estimation of sample size: $N=(t / d){ }^{2 *}(1-p) / p(t=1.96, p=0.127$ and $d=0.1)$ [1], we defined sample sizes of urban and rural groups. The sizes were 1800 and 2200 based on the ratio of urban and rural populations of the area, respectively, and the total was 4000 (more than the value $\mathrm{N}$ ).

Inclusion criteria: at least 18 years of age, had lived in the same area for more than 10 years, was selected from the census list according to the sampling method. Exclusion criteria: presence of abnormal autoantibodies, ceruloplasmin, and iron tests, consumption of more than $40 \mathrm{~g}$ of alcohol per day.

In the end, 3920 people responded and agreed to participate in the study, and their serum samples, demographic information, and behavioral factors were collected.

The response rate was high $(98 \%, 3920 / 4000)$. When we analyzed the relationship between abnormal liver tests and the risk factor, we excluded 75 people who had abnormal autoantibodies, ceruloplasmin, and iron tests. They consisted of 30, 29, and 16 people, respectively. In addition, 54 people who reported consuming at least $40 \mathrm{~g}$ of alcohol per day were excluded. In the end, 3791 people (3920-75-54=3791)(1800 men and 1991 women) were eligible for our analyses. 


\section{Data and blood collection}

The study team consisted of physicians and nurses who were trained in the survey methods in order to standardize the data collection, interviews, blood drawing and handling of serum samples. The selected participants were asked to fast overnight $(\geq 8$ h) and attend a local health center for their scheduled appointments. The selected subjects were visited at home if they could not attend the local health center. An interview using the structured questionnaire was conducted at the time of the participant's visit. The questionnaire included the following questions: (1) Do you sleep well? (2) Do you smoke? (number of cigarettes per day)? (3) Do you drink alcohol (number and type of drinks per day)? (4) Date of birth, sex, place of residence. Information on demographics (date of birth, sex, place of residence,) and behavioral factors (drinking, smoking, sleep quality) was obtained. The study protocol was approved by the Institutional Review Board of the First Hospital of JiLin University. After written informed consent was obtained, blood samples were taken from each participant for seroprevalence analyses. Sera were stored at $-20^{\circ} \mathrm{C}$ until tested at the First Hospital of JiLin University. Anthropometric measurements including height, weight, and waist and hip circumference were conducted by well-trained examiners on individuals wearing light clothing. Waist circumference was measured to the nearest $0.1 \mathrm{~cm}$ at the midpoint between the lower borders of the rib cage and the iliac crest. Abdominal ultrasonography was performed to detect the presence of fatty infiltration in the liver by physicians specializing in diagnostic imaging. All of whom used standard criteria in evaluating the images for hepatic fat [13]. A portable GE Logiq Book ultrasound set (GE Yokogawa Medical System, Tokyo, Japan) with a 3.8-MHz transducer was used. Fatty liver was diagnosed using ultrasonography with characteristic findings of diffusely increased liver echogenicity, as evidenced by: liver-kidney contrast, blurring of the intrahepatic vessels and the diaphragm caused by diffusely increased liver echogenicity, brightness of hepatic echogenicity with loss echoes of the posterior hepatic segments and the intrahepatic vessels, or invisibility of the diaphragm. Fatty liver was diagnosed by concurrence of 3 ultrasonographers, who were unaware of the subjects' clinical and biochemical status.

\section{Serological tests}

HBsAg, HCV antibody, autoantibodies, ceruloplasmin, iron studies, glucose, total cholesterol, LDL-cholesterol, HDL-cholesterol, triglyceride, ALT, AST, and GGT were assayed by standard methods with kits from Ke Hua (Shanghai, China). Blood cell counts were undertaken using an automatic cell counter (MEK-6318, Japan). All laboratory analyses were performed at the First Hospital of Jilin University.

\section{Definitions of the abnormal liver tests, metabolic syndrome and NAFLD}

Abnormal liver tests, ALT $\geq 45$ IU/L and/or GGT $\geq 50 \mathrm{IU} / \mathrm{L}$ and/or AST $\geq 45 \mathrm{IU} / \mathrm{L}$ and/or platelet counts $\leq 130 \times 10^{9} / \mathrm{L}$. These values are consistent with the upper or lower limits of normal established by the reference laboratory for the area. Cases of Wilson's disease, autoimmune hepatitis, elevated serum ferritin, saturated serum transferrin, and hereditary hemochromatosis were excluded. The metabolic syndrome was defined according to the National Cholesterol Education Program Adult Treatment Panel III (NCEP-ATP III, 2005), in which central obesity is an essential parameter (BMI $\geq 25)$ [14]. A modified waist circumference cutoff of $>90 \mathrm{~cm}$ was used in men and $>80 \mathrm{~cm}$ in women [15].

The metabolic syndrome was then defined as any individual who had two or more of the four following: 1) high blood pressure (>130/85 $\mathrm{mmHg}$ ) or anti-hypertensive medication, 2) elevated fasting blood glucose $(>5.6 \mathrm{mmol} / \mathrm{L})$ or anti-diabetic medication, 3) hypertriglyceridemia (>1.7 $\mathrm{mmol} / \mathrm{L})$, and 4$)$ low HDL-cholesterol (men, $<1.04 \mathrm{mmol} / \mathrm{L}$; women, $<1.3 \mathrm{mmol} / \mathrm{L}$ ).

Individuals meeting the following conditions were defined as having NAFLD: 1) consuming less than $40 \mathrm{~g}$ of alcohol per week, 2) having negative test for HBsAg or hepatitis C, and 3) having fat in the liver by ultrasound imaging.

Excessive alcohol intake was defined as alcohol intake $>40 \mathrm{~g}$ per day for one or more consecutive years. The cause of the abnormal liver tests was defined as unexplained when there was no evidence of fatty liver by ultrasonography, no excessive alcohol intake, no HBsAg or anti-HCV, and no other known causes of liver damage.

\section{Statistical analysis}

Statistical analyses were performed using SAS software (version 8.0). Clinical and biochemical characteristics were compared between men and women using Chi-Square test or Fisher's exact test. Logistic regression analysis was used to evaluate relationships between abnormal LTs (case group) and normal LTs (control group) in the entire survey population. Logistic regression analysis was also used to evaluate relationships between abnormal LTs (case group) and diseased with normal LTs (disease control group) in 
NAFLD, unexplained cases, HBV, HCV, or metabolic syndrome subgroups. The independent variables were age, gender, sleep quality, smoking, BMI, fasting plasma glucose, total cholesterol, triglyceride, HDL, and LDL. $\mathrm{P}<0.05$ was considered statistically significant.

\section{Results}

\section{Prevalence and etiology of abnormal liver tests}

A total of 3791 serum samples were collected from 1800 males, and 1991 females in the age group 18-79 years. Their median age was 43 years; $32.3 \%$ were between 18 and 39, 60.5\% between 40 and 64, and only $7.02 \%$ were over $65 ; 1773$ came from urban, and 2018 from rural areas.

The median ALT, AST, GGT, and platelet counts in patients without abnormal LTs were $16 \mathrm{U} / \mathrm{L}, 19.5$ $\mathrm{U} / \mathrm{L}, 16.8 \mathrm{U} / \mathrm{L}$, and $241^{*} 10^{9} / \mathrm{L}$, respectively; they were $41.4 \mathrm{U} / \mathrm{L}, 30.1 \mathrm{U} / \mathrm{L}, 69.9 \mathrm{U} / \mathrm{L}$, and $203 \mathrm{X} \mathrm{10}$ / L, respectively, in the abnormal LTs group. Table 1 summarizes the prevalence of abnormal liver tests according to etiology and age. The overall prevalence of abnormal liver tests was $14.77 \%$. The noticeable cause of abnormal liver tests was excess alcohol consumption alone $(18.21 \%)$, NAFLD alone $(11.61 \%)$, metabolic syndrome alone $(25 \%)$, both NAFLD and metabolic syndrome $(22.14 \%)$.

The prevalence first increased and then decreased progressively with increased age. Age was also the main factor contributing to NAFLD alone and metabolic syndrome alone as well as HBV infection and alcohol intake. Nevertheless, the prevalence of abnormal liver tests caused by $\mathrm{HCV}$ infection increased progressively with age. Abnormal liver tests caused by both NAFLD and metabolic syndrome were predominantly observed in the age group 40-64 years old. The study showed that both NAFLD and metabolic syndrome were potentially important etiologies of presumed liver injury (3.27\%). Etiologies such as alcohol intake alone and metabolic syndrome alone were more frequent, and NAFLD alone was less frequent in men. The abnormal liver tests occurred more frequently in men than in women (416 out of 1739 subjects, $23.92 \%$ vs. 144 out of 2052 subjects, $7.02 \%$; $\mathrm{P}<0.01$ ) (Table 2).

\section{Factors associated with abnormal liver tests}

Table 3 shows the results of individual risk factors and their association with abnormal liver tests. The overall prevalence of abnormal liver tests was $14.77 \%$ (560 out of 3791 subjects). The factors associated with abnormal liver tests were male gender, mid-old age, poor quality of sleep, smoking, alcohol intake, higher fasting plasma glucose, BMI, high total cholesterol, hypertriglyceridemia, and elevated LDL. However, except higher total cholesterol, other risk factors were significantly associated with the development of abnormal liver tests in a multiple logistic regression analysis.

Table I. Prevalence of Abnormal Liver Tests Classified by Etiology and Age

\begin{tabular}{lllll}
\hline Etiological factor & \multicolumn{2}{l}{ Age (years) } & Total $(\mathrm{n}=3791)$ \\
\cline { 2 - 5 } & $18 \sim 39(\mathrm{n}=1228) \mathrm{n}(\%)$ & $40 \sim 64(\mathrm{n}=2297) \mathrm{n}(\%)$ & $\geq 65(\mathrm{n}=266) \mathrm{n}(\%)$ & $\mathrm{n}(\%)(95 \% \mathrm{CI})$ \\
\hline Alcohol alone & $22(1.79)$ & $78(3.4)$ & $2(0.75)$ & $102(2.69)(2.17-3.20)$ \\
HBV $( \pm$ alcohol $)$ & $9(0.73)$ & $47(2.05)$ & $3(1.13)$ & $59(1.56)(1.16-1.95)$ \\
HCV $( \pm$ alcohol) & $4(0.33)$ & $10(0.44)$ & $3(1.13)$ & $17(0.45)(0.23-0.66)$ \\
HBV and HCV $( \pm$ alcohol $)$ & $0(0)$ & $2(0.09)$ & $0(0)$ & $2(0.05)(0.01-0.12)$ \\
NAFLD alone & $20(1.63)$ & $40(1.74)$ & $5(1.88)$ & $65(1.71)(1.3-2.12)$ \\
MS alone & $41(3.34)$ & $91(3.96)$ & $8(3.01)$ & $140(3.69)(3.09-4.29)$ \\
NAFLD and MS & $30(2.44)$ & $86(3.74)$ & $8(3.01)$ & $124(3.27)(2.7-3.8)$ \\
Unexplained & $16(1.3)$ & $34(1.48)$ & $1(0.38)$ & $51(1.35)(0.97-1.71)$ \\
Total $n(\%)(95 \% \mathrm{CI})$ & $142(11.56)(9.77-13.35)$ & $388(16.89)(15.35-18.42)$ & $30(11.28)(7.47-15.1)$ & $560(14.77)(13.6-15.9)$ \\
\hline
\end{tabular}

Abnormal liver tests: ALT $\geq 45 \mathrm{IU} / \mathrm{L}$ and/or GG T $\geq 50 \mathrm{IU} / \mathrm{L}$ and/or AST $\geq 45 \mathrm{IU} / \mathrm{L}$ and/or platelet counts $\leq 130 \mathrm{X} 10^{9} / \mathrm{L}$; CI, confidence interval; NAFLD, non-alcoholic fatty liver; alcohol alone, alcohol intake $\geq 28 \mathrm{~g} / \mathrm{d}$ for more than two years; there were no smoking alone or poor sleep quality alone groups.

NAFLD was defined by fatty appearing liver on ultrasound with HBsAg (-), anti-HCV Ab (-), no excessive alcohol consumption, and no other known etiologies of liver disease. 
Table 2. Causes for Abnormal Liver Tests Classified by Gender

\begin{tabular}{lllll}
\hline Etiological factor & Male $(\mathrm{n}=416) \mathrm{n}(\%)$ & Female $(\mathrm{n}=144) \mathrm{n}(\%)$ & Total $(\mathrm{n}=560) \mathrm{n}(\%)$ & $\mathrm{P}$ value \\
\hline Alcohol alone & $102(24.51)$ & $0(0)$ & $102(18.21)$ & $<9(10.54)$ \\
HBV $( \pm$ alcohol $)$ & $44(10.57)$ & $15(10.41)$ & $17(3.04)$ & $<0.05$ \\
HCV $( \pm$ alcohol $)$ & $9(2.16)$ & $8(5.55)$ & $2(0.36)$ & $<0.05$ \\
HBV and HCV $( \pm$ alcohol $)$ & $1(0.24)$ & $1(0.69)$ & $65(11.61)$ & $<0.05$ \\
NAFLD alone & $38(9.13)$ & $27(18.75)$ & $140(25)$ & $<0.05$ \\
MS alone & $122(29.32)$ & $18(12.5)$ & $124(22.14)$ & $<0.05$ \\
NAFLD and MS & $70(16.82)$ & $54(37.5)$ & $51(9.11)$ & $<0.05$ \\
Unexplained & $30(7.21)$ & $21(14.58)$ & $560(100)$ & $<0.05$ \\
Total $n$ (\%) & $416(100)$ & $144(100)$ & &
\end{tabular}

HBV, hepatitis B virus; $\mathrm{HCV}$, hepatitis C virus; ALT, alanine aminotransferase; Abnormal liver tests: ALT $\geq 45$ IU/L and/or GG T $\geq 50$ IU/L and/or AST $\geq 45 \mathrm{IU} / \mathrm{L}$ and/or platelet counts $\leq 130 \times 10^{9} / \mathrm{L}$; CI, confidence interval; NAFLD, non-alcoholic fatty liver; alcohol alone, alcohol intake $\geq 28 \mathrm{~g} / \mathrm{d}$ for more than two years; there were no smoking alone or poor sleep quality alone groups.

NAFLD was defined by fatty appearing liver on ultrasound with HBsAg (-), anti-HCV Ab (-), no excessive alcohol consumption, and no other known etiologies of liver disease.

Table 3. Association of Risk Factors with Individuals With or Without Abnormal Liver Tests by Multiple Analyses

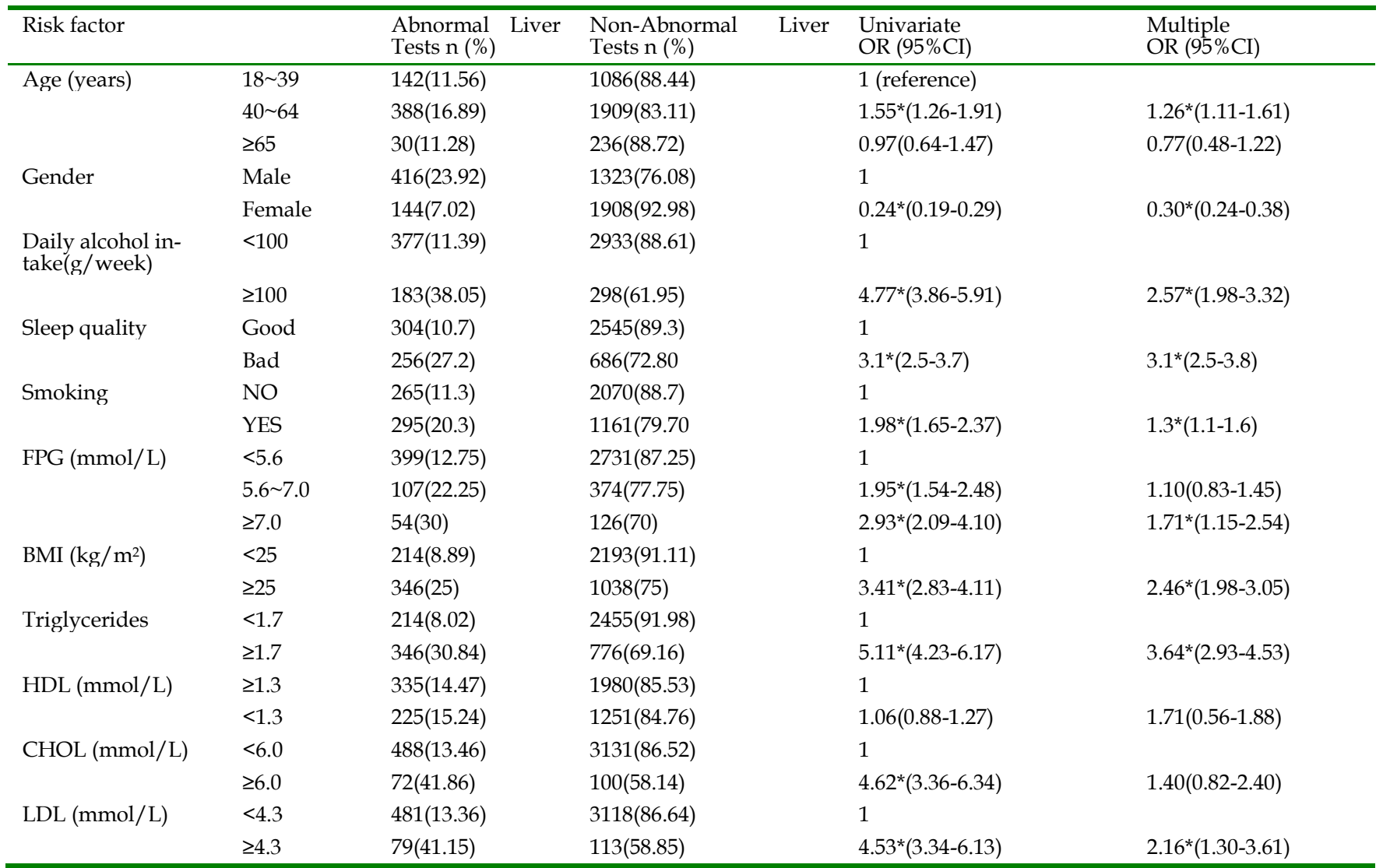

BMI, body mass index; CI, confidence interval; FPG, fasting plasma glucose; HDL, high density lipoprotein; LDL, low density lipoprotein; $\mathrm{CHOL}$, total cholesterol;

$* \mathrm{P}<0.001$ vs. reference. 
Factors associated with abnormal LTs in individuals with NAFLD, metabolic syndrome, $H B V$, $\mathrm{HCV}$ or unexplained subgroups

We found that male gender, poor quality of sleep, smoking and younger age with increased triglyceridemia, FPG and BMI were associated with abnormal LTs in individuals with NAFLD in multivariate analyses. Meanwhile, male gender, alcohol intake, higher triglyceridemia, FPG and LDL were associated with abnormal LTs in individuals with metabolic syndrome. The prevalence of HBV and an-
ti-HCV antibody was 5\% (190/3791) and $1.1 \%$ (45/3791). Abnormal LTs were associated with male gender, older age, and high triglycerides in $\mathrm{HBV}$-positive individuals, but not in anti-HCV antibody-positive individuals. Abnormal LTs in HBVand HCV-positive individuals was less associated with biochemical or metabolic factors. Male gender and high triglyceridemia were associated with individuals with abnormal LTs of unexplained cause (Table 4).

Table 4. Multiple Logistic Regression Analysis of Risk Factors Associated with Abnormal Liver Tests in Different Diseases

\begin{tabular}{|c|c|c|c|c|c|c|}
\hline & & HBV group & $\mathrm{HCV}$ group & MS group & NAFLD group & $\begin{array}{l}\text { Unexplained } \\
\text { group }\end{array}$ \\
\hline$\%(\text { case } / \text { total })^{*}$ & & $32.1(61 / 190)$ & $42(19 / 45)$ & $32.85(264 / 814)$ & $31.7(189 / 596)$ & $2.37(51 / 2155)$ \\
\hline Risk factor & & Abnormal Liver 1 & odds ratio $(95$ & ence interval) & & \\
\hline \multirow[t]{3}{*}{ Age (years) } & $18 \sim 39$ & 1(reference) & & & 1 & \\
\hline & $40 \sim 64$ & $6.33^{*}(4.69-8.90)$ & & & $0.63(0.39-1.02)$ & \\
\hline & $\geq 65$ & $11.61^{*}(8.41-15.41)$ & & & $0.40^{*}(0.18-0.91)$ & \\
\hline \multirow[t]{2}{*}{ Gender } & Male & 1 & & 1 & 1 & 1 \\
\hline & Female & $0.37^{*}(0.17-0.82)$ & & $0.22^{*}(0.15-0.33)$ & $0.24^{*}(0.16-0.36)$ & $0.46^{*}(0.26-0.67)$ \\
\hline \multirow{2}{*}{$\begin{array}{l}\text { Daily alcohol in- } \\
\text { take(g/week) }\end{array}$} & $<100$ & & & 1 & & \\
\hline & $\geq 100$ & & & $1.77^{*}(1.42-2.80)$ & & \\
\hline \multirow[t]{2}{*}{ Sleep quality } & Good & & & & 1 & \\
\hline & Bad & & & & $26^{*}(12-59)$ & \\
\hline \multirow[t]{2}{*}{ Smoking } & No & & & & 1 & \\
\hline & Yes & & & & $2.5^{*}(1.2-5)$ & \\
\hline \multirow[t]{3}{*}{$\mathrm{FPG}(\mathrm{mmol} / \mathrm{L})$} & $<5.6$ & & & 1 & 1 & \\
\hline & $5.6 \sim 7.0$ & & & $0.90(0.61-1.33)$ & $1.13(0.68-1.88)$ & \\
\hline & $\geq 7.0$ & & & $1.45^{*}(1.25-2.48)$ & $1.40 *(1.14-2.65)$ & \\
\hline \multirow[t]{2}{*}{$\mathrm{BMI}\left(\mathrm{kg} / \mathrm{m}^{2}\right)$} & $<25$ & & & & 1 & \\
\hline & $\geq 25$ & & & & $1.69^{*}(1.42-2.79)$ & \\
\hline \multirow{2}{*}{$\begin{array}{l}\text { Triglyceride } \\
(\mathrm{mmol} / \mathrm{L})\end{array}$} & $<1.7$ & 1 & & 1 & 1 & 1 \\
\hline & $\geq 1.7$ & $2.77^{*}(1.89-3.07)$ & & $1.95^{*}(1.32-2.88)$ & $2.26^{*}(1.52-3.35)$ & $5.12^{*}(3.47-7.60)$ \\
\hline \multirow[t]{2}{*}{$\mathrm{LDL}(\mathrm{mmol} / \mathrm{L})$} & $<4.3$ & & & 1 & & \\
\hline & $\geq 4.3$ & & & $2.96^{*}(1.8-3.81)$ & & \\
\hline
\end{tabular}

HBV, hepatitis B virus; HCV, hepatitis C virus; MS, metabolic syndrome; BMI, body mass index; FPG, fasting plasma glucose; HDL, high density lipoprotein; LDL, low density lipoprotein; $\mathrm{CHOL}$, total cholesterol; NAFLD, non-alcoholic fatty liver; alcohol alone; ${ }^{*} \mathrm{P}<0.001 \mathrm{vs}$. the reference.

$\%$ (case/total), *percent of abnormal liver tests in various disease subgroups.

\section{Discussion}

Based on earnings in the middle of the income range of most of the inhabitants, and the sex and age distribution, at the level of life, economic and cultural development in the province, Dehui City is representative of JiLin in general. A multistage, tiered-system sampling method used to control selec- tion bias provided a study sample that was a good representative of the target population in JiLin.

The population in our study had dietary habits that are different from those of populations in southern China and other industrialized areas. In the present study, we found a very high prevalence of abnormal liver tests in the adult population $(14.77 \%)$ and elevated serum aminotransferase levels (11.1\%). 
In previous community-based studies in Italy, the percentage of individuals with persistently abnormal LTs was $12.7 \%$ [1]. Data from the Third National Health and Nutrition Examination Survey, which was conducted in the United States from 1988 to 1994, indicated that $7.9 \%$ of adults had elevated serum aminotransferase levels [10]. The prevalence of elevated ALT was reported to be $11 \%$ in Taiwan [12]. Therefore, the observed prevalence of $14.7 \%$ of abnormal liver tests or elevated serum aminotransferase levels in the current study in Jilin, China was relatively high.

This percentage would have been lower if the upper limits of normal for aminotransferases were higher. However, the public health and clinical utility of altering them is debatable [16]. The causes of abnormal liver tests may vary in different geographic areas. The factors associated with abnormal liver tests were male gender, old age, alcohol intake, poor quality of sleep, smoking and metabolic factors (e.g., hypertriglyceridemia), which confirms data in previous studies $[1,12]$. Metabolic factors accounted for most of the cases of abnormal liver tests in present study.

ALT is more specific for active hepatocellular damage. The prevalence of elevated AST reflects the incidence of other conditions besides liver damage. Therefore, it is not surprising that the combination of AST and thrombocytopenia would be higher than ALT and thrombocytopenia. An epidemiological survey published in 2000 revealed that $10.74 \%$ of the Chinese population had positive hepatitis B surface (HBsAg), indicating that China is one of the highest endemic regions of HBsAg in the world [17]. The prevalence of $\mathrm{HCV}$ was low in China, with one report showing only a 3\% prevalence rate in the Shaanxi Province, China [18]. Hepatitis viral infection is not the most common cause of chronic liver disease in Jilin, China. We found that the prevalence of HBsAg carriers was 5\%, and that it decreased with older age, which would currently make the region to be considered as an intermediate endemic region. ${ }^{19-21}$

In addition, our data on HBsAg positivity $(190 / 3791=5 \%)$ were more than two-fold lower than that of $10.7 \%$ in the pre-vaccination era (Table 4 ). Ten years ago, the seroprevalence of positive-HBsAg was $7 \%, 8 \%$, and $9 \%$ in the Shaanxi, Shangdong, and Jilin Provinces, respectively [18, 22-23]. In the 65 and older age group, abnormal liver tests attributable to HBV were relatively low. Explanations for this include the known increase in spontaneous seroconversion with duration of infection, and a known tendency of HBV viral load to decline with duration of infection. In addition, progressive mortality due to HBV-related sequelae may have led to a decreased prevalence of individuals with $\mathrm{HBV}$-infection in the 65 and older age group [24]. In contrast, the prevalence of anti-HCV antibody was $1.1 \%$, and the risk significantly increased with older age. The current data are consistent with those of other studies [25]. Adult immunization for $\mathrm{HBV}$ is an effective means to decrease the seroprevalence of HBsAg, but there is no clear explanation for the observations on HCV seroprevalence.

The prevalence rates of metabolic syndrome and NAFLD in the abnormal LTs group were different between the genders. It is not clear whether gender is an independent risk factor for them [26]. Insulin resistance is considered to be a common denominator of NAFLD because insulin resistance is very common in obesity, and hypertriglyceridemia, and is the hallmark of type 2 diabetes mellitus [27]. The pathogenesis of NAFLD is hypothesized to be the 'two-hit theory' and is related to metabolic syndrome $[1,4]$. The first hit consists of steatosis induced by insulin resistance, and the second hit consists of oxidative stress in mitochondria of lipid-laden hepatocytes. When fatty liver is associated with necroinflammation and fibrosis [28], NAFLD can progress to cirrhosis and hepatocellular carcinoma [29]. The results from our current study may reflect the Westernization of dietary habits and sedentary lifestyles in this population. These data are also consistent with literature suggesting that metabolic syndrome is a predictor of fibrosis and has an independent pathogenic role in NAFLD [30]. The apparently higher prevalence of NAFLD in this population compared to previous reports probably reflects the fact that the population is rapidly changing towards increasing obesity.

Male gender and metabolic factors (e.g., triglyceridemia, body mass index, and low density lipoprotein) were risk factors for abnormal LTs of unexplained cause in previous surveys from a Mediterranean town and Taiwan [1, 12]. Medications such as anti-hypertensives, lipid-lowering, or anti-diabetic drugs were other possible causes of abnormal liver tests. When these were excluded in the present study, the risk factors were also associated with abnormal LTs in individuals with unexplained causes. A reason for the cases of unexplained causes may have been the inclusion of cases of early stage diseases which were not diagnosed due to a lack of signs and symptoms (e.g., metabolic syndrome).

Our present study showed that metabolic factors (e.g., triglyceridemia, body mass index, and low density lipoprotein) were less associated with abnormal LTs in individuals with HBV or HCV infection with the exception of gender in the regression models. The reason for this may be that the number of people with $\mathrm{HBV}$ and $\mathrm{HCV}$ was low. In a multiple logistic regres- 
sion model, generally there should be ten events for each variable. The abnormal LTs in individuals with HBV or HCV may have been due to the viral infections [31]. Poor quality of sleep, and smoking were associated with abnormal liver tests in individuals with NAFLD and the other groups, suggesting that those factors may promote abnormal LTs in individuals with NAFLD and the other groups. Cessation of smoking and decreasing alcohol intake may ameliorate liver damage.

One of the limitations in the present study is that the diagnosis of fatty liver was based on ultrasound imaging, which was not confirmed by liver biopsy, the gold standard for the assessment of liver histology and a key test to diagnose NAFLD.

\section{Conclusion}

The prevalence of HBV and HCV was found to be lower than previously reported. Therefore, abnormal LTs contributed by viral infections are not likely to be as significant in this geographic area as in the past. In individuals with abnormal LTs, metabolic syndrome alone, NAFLD alone, and alcohol intake were found to be important etiological factors, especially both MS and NAFLD. The estimated prevalence and etiologies of abnormal LTs may be useful in implementing strategies for reducing the potential burden of CLD.

\section{Abbreviations}

CLD: chronic liver disease; HBV: hepatitis B virus; HCV: hepatitis $C$ virus; NAFLD: nonalcoholic fatty liver disease; ALT: alanine aminotransferase; AST: aspartate aminotransferase; GGT: gamma glutamyltransferase; LTs: liver tests; BMI: body mass index; FPG: fasting plasma glucose; HDL: high density lipoprotein; LDL: low density lipoprotein; CHOL: total cholesterol.

\section{Conflict of Interest}

The authors declare that they have no conflict of interests.

\section{Acknowledgments}

We sincerely thank the participants who contributed to this work and the survey staff at the First Hospital of Jilin University.

\section{References}

1. Pendino GM, Mariano A, Surace $P$, et al. Prevalence and etiology of altered liver tests: a population-based survey in a Mediterranean town. Hepatology. 2005;41:1151-9.
2. Bugianesi E, Zannoni C, Vanni E, et al. Nonalcoholic fatty liver and insulin resistance: a cause-effect relationship. Dig Liver Dis. 2004;36:165-73.

3. Flegal KM, Carroll MD, Ogden CL, et al. Prevalence and trends in obesity among US adults, 1999-2008. JAMA. 2010;303:235-41.

4. Marchesini G, Bugianesi E, Forlani G, et al. Nonalcoholic fatty liver, steatohepatitis, and the metabolic syndrome. Hepatology. 2003;37:917-23.

5. Wang Y, Dong J, Liu HY, et al. Biochemical characteristics and risk factors in non-alcoholic fatty liver. Front Biosci. 2010;2:105-10.

6. Kelishadi R, Cook SR, Amra B, et al. Factors associated with insulin resistance and non-alcoholic fatty liver disease among youths. Atherosclerosis. 2009;204:538-43.

7. Strauss RS, Barlow SE, Dietz WH. Prevalence of abnormal serum aminotransferase values in overweight and obese adolescents. J Pediatr. 2000;136:727-33.

8. Daniel S, Ben-Menachem T, Vasudevan G, et al. Blumenkehl M. Prospective evaluation of unexplained chronic liver transaminase abnormalities in asymptomatic and symptomatic patients. Am J Gastroenterol. 1999;94:3010-4.

9. Katkov WN, Friedman LS, Cody H, et al. Elevated serum alanine aminotransferase levels in blood donors: the contribution of hepatitis $\mathrm{C}$ virus. Ann Intern Med. 1991;115:882-4.

10. Clark JM, Brancati FL, Diehl AM. The prevalence and etiology of elevated aminotransferase levels in the United States. Am J Gastroenterol. 2003;98:960-7.

11. Fang J, Zhang JP, Luo CX, et al. Carotid Intima-media thickness in childhood and adolescent obesity relations to abdominal obesity, high triglyceride level and insulin resistance. Int J Med Sci. 2010;7:278-83.

12. Chen $\mathrm{CH}$, Huang $\mathrm{MH}$, Yang JC, et al. Prevalence and etiology of elevated serum alanine aminotransferase level in an adult population in Taiwan. J Gastroenterol Hepatol. 2007;22:1482-9.

13. Sanyal AJ. AGA technical review on nonalcoholic fatty liver disease. Gastroenterology. 2002;123:1705-25.

14. Alberti KG, Zimmet P, Shaw J. Metabolic syndrome--a new world-wide definition. A Consensus Statement from the International Diabetes Federation. Diabetic med. 2006;23:469-80.

15. WHO/IASO/IOTF. The Asia-Pacific perspective: redefining obesity and its treatment. Melbourne: Health Communications Australia. 2000

16. Prati D, Taioli E, Zanella A, et al. Updated definitions of healthy ranger for serum alanine aminotransferase levels. Ann Intern Med. 2002;137:1-9.

17. Wen YM. Laboratory diagnosis of viral hepatitis in China: the present and the future. Clin Chem Lab Med. 2001;39:1183-9.

18. Shimbo S, Zhang ZW, Gao WP, et al. Prevalence of hepatitis B and $C$ infection markers among adult women in urban and rural areas in Shaanxi Province, China. Southeast Asian J Trop Med Public Health. 1998;29:263-8.

19. Chao J, Chang ET, So SK. Hepatitis B and liver cancer knowledge and practices among healthcare and public health professionals in China: a cross-sectional study. BMC Public Health. 2010;10:98-102.

20. Kurugöl Z, Koturoğlu G, Akşit S, et al. Seroprevalence of hepatitis $B$ infection in the Turkish population in Northern Cyprus. Turk J Pediatr. 2009;51:120-6.

21. Zhang ZW, Shimbo S, Qu JB, et al. Hepatitis B and C virus infection among adult women in Jilin Province, China: an urban-rural comparison in prevalence of infection markers. Southeast Asian J Trop Med Public Health. 2000;31:530-6.

22. Zhang LP, Yang P, Li FH, et al. Hepatitis viruses infection situation in Mianyang of the Sichuan province. Zhonghua Shi Yan He Lin Chuang Bing Du Xue Za Zhi. 2008;22:449-51. 
23. Shimbo S, Zhang ZW, Qu JB, et al. Urban-rural comparison of $\mathrm{HBV}$ and $\mathrm{HCV}$ infection prevalence among adult women in Shandong Province, China. Southeast Asian J Trop Med Public Health. 1997;28:500-6.

24. Chu CM, Lian YF. HBsAg seroclearance in asymptomatic carriers of high endemic areas: appreciably high rates during a long-term follow-up. Hepatology. 2007;45:1187-92

25. Wang CS, Chang TT, Chou P. Differences in risk factors for either a hepatitis B carrier or anti-hepatitis C in a hepatoma-hyperendemic area in rural Taiwan. Clin Epidemiol. 1998;51:733-8.

26. Feldman M, Friedman L S, Brandt L J. Nonalcoholic fatty liver disease. Gastrointestinal and Liver Disease.8th ed. Canada: Saunders; 2006: 1793-1805.

27. Chitturi S, Abeygunasekera S, Farrell GC, et al. NASH and insulin resistance: insulin hypersecretion and specific association with the insulin resistance syndrome. Hepatology. 2002;35:373-9.

28. Brunt EM. Nonalcoholic steatohepatitis. Semin Liver Dis. 2004;24:3-20.

29. Bugianesi E, Leone N, Vanni E, et al. Expanding the natural history of nonalcoholic steatohepatitis: from cryptogenic cirrhosis to hepatocellularcarcinoma. Gastroenterology. 2002;123:134-40.

30. Bugianesi E, Manzini P, D'Antico S, et al. Relative contribution of iron burden, HFE mutations, and insulin resistance to fibrosis in nonalcoholic fatty liver. Hepatology. 2004;39:179-87.

31. NORIO HAYASHI, TATSUYA KANTO, TETSUO TAKEHARA. Immunopathogenesis of type $C$ hepatitis: dendritic cell in HCV infection. J Gastroenterol Hepatol. 2004;19:s84-s7. 